\title{
À la recherche du temps vécu
}

Le théâtre de la Socìetas Raffaello Sanzio

In Search of Lived Time. The Theatre of the Societas Raffaello Sanzio?

\section{Belén Tortosa Pujante}

\section{(2) OpenEdition}

\section{Journals}

Édition électronique

URL : https://journals.openedition.org/cher/4587

DOI : $10.4000 /$ cher.4587

ISSN : 2803-5992

Éditeur

Presses universitaires de Strasbourg

\section{Édition imprimée}

Date de publication : 30 novembre 2021

Pagination : 185-196

ISBN : 979-10-344-0095-9

ISSN : 1968-035X

\section{Référence électronique}

Belén Tortosa Pujante, «À la recherche du temps vécu », reCHERches [En ligne], 27 | 2021, mis en ligne le 30 novembre 2021, consulté le 12 janvier 2022. URL : http://journals.openedition.org/cher/4587 ; DOI : https://doi.org/10.4000/cher.4587

\section{(c) (i) (2)(2)}

Ce(tte) œuvre est mise à disposition selon les termes de la Licence Creative Commons Attribution Pas d'Utilisation Commerciale - Partage dans les Mêmes Conditions 4.0 International. 


\title{
À la recherche du temps vécu Le théâtre de la Societas Raffaello Sanzio
}

\author{
Belén Tortosa Pujante*
}

\section{Le tournant performatif comme un nouveau paradigme}

La représentation du temps est une chose inaccessible. Le temps échappe au flash d'une caméra ou au trait de pinceau sur une toile. C'est un impossible qui nous montre ce qui a été. En fait, dans l'histoire de l'art les relations entre temps et art ont été une constante: la nature morte de Philippe de Champaigne, Vanitas, du XVII siecle ou Le grand verre de Marcel Duchamp ont suscité différentes interprétations où nous pouvons trouver un dénominateur commun: le temps, représenté à partir de différents éléments, soit symboliques comme dans l'œuvre de Champaigne soit conceptuelles comme dans Le grand verre à travers la poussière. Cependant, nous pourrions dire que l'art de la performance et le théâtre sont les arts les plus proches de cet impossible. La rénovation théâtrale qui a eu lieu durant le $\mathrm{xx}^{\mathrm{e}}$ siècle, à travers le travail d'auteurs comme Meyerhold, Artaud, Gordon Craig, Grotowsky, et un long etcétéra, rend plus évidente cette proximité et nous fait comprendre le théâtral comme un fait non plus dépendant de la littérature dramatique et l'élève ainsi à la catégorie d'art scénique: avec une identité propre et une autonomie par rapport au littéraire.

Par exemple, Erika Fischer-Litche nous avertit du changement de paradigme qu'implique le tournant performatif dans les arts et plus concrètement dans le théâtre $\mathrm{du} \mathrm{xx}^{\mathrm{e}}$ siècle: l'œuvre n'est plus entendue comme un signe, mais comme un évènement vivant où tous ceux qui participent à l'événement partagent un même espace et un même temps. Chaque élément matériel ne se comporte plus comme un signifiant auquel on peut attribuer une signification. C'est la matérialité de l'événement qui déclenche un certain nombre de réactions psychologiques, affectives, volontaires et énergétiques qui à leur tour

* Belén Tortosa Pujante, Université Saint Jacques de Compostelle 
provoquent d'autres actions, dans un processus continu de feedback. Fischer situe le premier tournant performatif lors de la mise en place de recherches sur les rituels et les études théâtrales dans la culture européenne du $\mathrm{xx}^{\mathrm{e}}$ siècle - et non lors de l'émergence de la culture de la performance dans les années 1960 et 1970. Au début du $\mathrm{xx}^{\mathrm{e}}$ siècle apparaissent déjà les premières études théâtrales qui comprennent l'origine du théâtre grec et, par conséquent, du théâtre occidental, à partir du rituel et non du texte littéraire. Par exemple, Richard Schechner nous raconte ce lien avec le rituel à partir de l'étude des travaux des anthropologues Van Gennep et Victor Turner. Pour Richard Schechner, le processus rituel permet d'ouvrir un espace et un temps favorables à ce qu'il qualifie "anti-structure, a setting aside of restraints, a suspensión of social rules or the temporary adherence to an alternative set of rules ${ }^{1} »$ (2002:88). De même, la nature performative du rituel a imposé une nouvelle conception spatiotemporelle de la scène, comprise comme un événement ou un processus et non comme une œuvre ou un résultat final. Jerzy Grotowski, fondateur du théâtre de laboratoire polonais et l'une des principales icônes du théâtre rituel ou de ce qu'il a appelé le «théâtre pauvre», a déclaré ce qui suit:

The theatre, when it was still part of religion, was already theatre: it liberated the spiritual energy of the congregation or tribe by incorporating myth and profaning or rather transcending it. The spectator thus had a renewed awareness of his personal truth in the truth of the myth, and through fright and a sense of the sacred he came to catharsis ${ }^{2}$. (Grotowski, 2002: 22-23)

Cette idée (lien théâtre-rituel) contribue de manière décisive à soutenir le concept de tournant performatif où l'attention se déplace vers les relations établies entre acteurs et spectateurs. Dans le contexte de cette redéfinition relationnelle, un concept clé comme la représentation commence à être remis en question. Le théâtre est alors conçu comme la possibilité que quelque chose se passe entre acteurs et spectateurs, et non plus comme la représentation d'un monde fictif.

Le travail artistique de la Societas Raffaello Sanzio est un exemple de ce changement de paradigme. De ses débuts dans les années 1980 à aujourd'hui son théâtre s'inscrit dans une tradition artistique éloignée d'un logocentrisme caractéristique dans lequel le texte dramatique reste l'axe prédominant. Il plaide d'une part pour un métissage des différents arts, tels que les arts visuels, le cinéma, la musique, la vidéo, la danse ou la poésie, et met l'accent sur un discours philosophique, scientifique et historico-religieux de l'autre. Ces présupposés théoriques deviennent à leur tour, contemporains de certains

1 «d'anti-structure, de mise de côté des contraintes, de suspension de règles sociales ou d'adhésion temporaire à un ensemble alternatif de règles.»

2 «Lorsqu'il faisait encore partie de la religion, le théâtre était déjà du théâtre: il libérait l'énergie spirituelle de la congrégation ou de la tribu, incorporant le mythe et le profanant, ou plutôt le transcendant. Le spectateur a recueilli une nouvelle perception de sa vérité personnelle dans la vérité du mythe et à travers la terreur et le sentiment du sacré atteint la catharsis. » 
processus de transformation de la scène utilisés dans le théâtre occidental depuis la fin du $\mathrm{xx}^{\mathrm{e}}$ siècle jusqu'à aujourd'hui. Différents critiques ont donné à ces transformations le nom de théâtre postdramatique (Lehmann, 2013), performatif (Fischer-Lichte, 2011), ou postspectaculaire (Eiermann, 2012).

Dans l'ensemble du travail de la Socìetas, il est inévitable d'observer le fait qu'il réponde à une même question: celle qui vise un "art qui ne veut pas reproduire mais refaire le monde» (Claudia Castellucci et Romeo Castellucci, 2013: 9). L'esprit iconoclaste de la troupe perdure au-delà de ses représentations artistiques, à travers son travail pédagogique. À la manière des poètes symbolistes du XIX ${ }^{\text {e }}$ siècle, ils recherchent un shock, un obstacle à travers la destruction de l'expérience même, c'est-à-dire à travers la création de coordonnées spatiotemporelles différentes. À travers cette destruction, le "nouveau” ne se présente pas comme la recherche d'un nouvel objet de l'expérience, mais se vit telle une «éclipse» et une suspension temporelle de notre vision habituelle du monde.

La troupe italienne entreprend ainsi un "pèlerinage» dans les profondeurs de la matière scénique (il est ici important de comprendre le terme scénique dans une dimension qui dépasse les limites de la scène), afin de créer d'autres réalités et d'autres mondes alternatifs, de créer d'autres temps et espaces qui ne remplacent pas «l'œuvre de la nature», mais l'amplifient à travers de nouvelles créations. Au cours des quarante dernières années, son travail artistique a exploré différents langages et formes d'expression. Une recherche presque obsessionnelle $\mathrm{du}$ passage du mythe et du rituel vers la tragédie et la représentation, de sorte que son œuvre s'articule autour d'un triangle délimité par les trois sommets mythetragédie-réalité.

\section{Le temps du mythe et le temps de la représentation}

Qu'est-ce que cela signifie aujourd'hui d'entrer en relation avec le mythe? Quel chemin la Societas emprunte-t-elle dans sa recherche d'une nouvelle réalité «mythique»? Que cache la pensée mythique qui puisse rapprocher la compagnie de l'exploration de nouvelles formes de langage théâtral?

Le travail avec le mythe a permis de créer des réalités alternatives, fictives, construites sur la base de contradictions et de conflits insolubles qui échappent à la logique. La Societas a recherché la rupture de concepts clés de la scène théâtrale, comme le concept de représentation, pour tenter de trouver un langage iconoclaste. Cependant, cette rupture ne signifie pas qu'il faille aller au-delà de la raison, mais elle manifeste le besoin de trouver une image pour créer une nouvelle expérience qui, plutôt que d'être expliquée, sera révélée. Le travail de la troupe sur le mythe va au-delà de sa recréation. Il consiste à le réaliser (le rendre réel) à nouveau. Le théâtre de la Socìetas cherche à récupérer la force originelle présente dans la pensée mythique, non pas à partir du récit mythique, mais à travers l'expérience mythique. Ainsi, le corps sera privilégié à travers la danse, l'image, la peinture, la vidéo, la musique, etc. L'objectif est d'activer une imagination mythique chez le spectateur à travers la présence 
physique et tangible de chacun de ces éléments; se diriger vers les origines du théâtre presque par inadvertance à travers l'expérience du rite.

Dans un effort constant pour redonner vie au symbole, la Societas pratique la déstabilisation précise de la signification du symbole par l'utilisation du mythe. Il y a différents symboles dans le théâtre de Romeo Castellucci qui se répètent tout au long de ses spectacles comme s'il s'agissait d'une série de spores qui créent un réseau hypertextuel de signification lié à l'expérience mythique du théâtre. Tous ces symboles recherchent l'activation de la pensée mythique de la part du spectateur; une pensée qui s'incarne à travers la physicalité des corps sur scène, l'attention portée à leur mouvement, leur expression vocale, la musique ou le bruit, la lumière et l'atmosphère ; bref, vers la matérialité de la scène à la recherche d'un événement unique et irremplaçable qui illumine notre mémoire.

Quelques symboles importants sont présents dans l'œuvre de la Societas: symbole de la chute (catabase), de la mère (féminité) ou des forces telluriques, etc., dans le but de créer une nouvelle mythologie de la vision. Tous ces symboles subissent un processus de transfiguration (on peut voir le même symbole représenté à travers différentes figures ou formes) qui est utilisé comme technique ou procédure pour créer ce réseau hypertextuel.

Le rituel se présente comme un moment où le mythe se confronte à la fiction des corps des acteurs et au regard du spectateur, car «un rituel est la représentation d'un mythe. En participant à un rituel, vous participez à un mythe» (Campbell, 2015: 118). Pour reprendre les mots de Mircea Eliade, «en 'vivant' les mythes, on sort du temps profane (chronologique) et on aboutit à un temps qualitativement différent, un temps 'sacré', à la fois primordial et indéfiniment récupérable»(1973: 30). Le rite, et donc la représentation théâtrale, permet de sortir de ce temps quotidien, "profane», pour pénétrer dans un temps primordial, "sacré», de telle façon qu'il rappelle et rend contemporain le mythe primordial. Le rite travaille donc avec le mystère, lequel se développe comme un élément central dans la recherche de cette image ou symbole révélé.

\section{Le temps du tragique}

À travers ce travail de descente dans le ventre maternel et tellurique de notre existence et de l'existence du théâtre, une refonte du tragique dans notre société contemporaine est par ailleurs nécessaire pour la Societas. Nous rapprocher de ce second sommet du triangle implique fondamentalement de mettre en lumière le théâtre pré-tragique, lié à une présence féminine qui conserve le mystère de la création et de la mort de notre corps.

L'inclination de la troupe de Cesena pour le caractère pré-tragique du théâtre s'inscrit dans les réflexions nietzschéennes sur la tragédie, comprenant toutes les manifestations artistiques en termes de dualité apollinienne et dionysiaque. À cet égard, Hans-Thies Lehmann a souligné la relation particulière entre le théâtre post-dramatique actuel d'une saveur inévitablement postmoderne et le théâtre de la Grèce pré-tragique, lié aux rituels et aux fêtes orgiaques. Pour 
Lehmann, le théâtre contemporain évolue dans une référence continue vers ce qu'il appelle le «motif tragique» (2017:27). Au lieu de parler d'une continuité du drame tragique, Lehmann définit le motif tragique comme un mouvement de «transgression», qui a fait son apparition de manière instable à travers les âges.

Cette transgression se trouve présente dans la recherche d'un langage physiquement (charnel) tragique dans le théâtre de la Socìetas Raffaello Sanzio. Des épisodes comme Br.\#04 de Tragedia Endogonidia en sont le reflet: nous voyons l'image d'un homme dans un sac poubelle qui y a été introduit après avoir été torturé par des hommes déguisés en policiers. Ces derniers lui apportent un micro et l'homme commence à parler à l'intérieur du sac dans un langage incompréhensible. Le micro amplifie les sons de quelqu'un qui ne trouve plus les mots pour se faire comprendre, et qui devient un morceau de viande, présence pure du corps souffrant. On lui demande de parler à travers le micro, mais ce qu'il parvient à émettre ce sont les mots de sa propre respiration, les "mots-cris», les mots d'un «corps sans organes».

Cette même conception de la langue est perceptible à travers le traitement de l'alphabet latin. Au fil des différents épisodes, nous verrons la projection à grande vitesse de différentes lettres de l'alphabet latin, mélangées à des taches de Rorschach:

Un linguaggio al negativo, il suono del linguaggio reso in stracci e macerie, che disperatamente ed urgentemente, continua a portare un qualcosa che deve essere comunicato ${ }^{3}$. (Ridout, 2002: 9)

De cette manière, la nouvelle communauté tragique ne se crée pas autour de la parole, mais naît dans l'impossibilité de communiquer, dans le silence:

La creazione della tragedia è per noi un passaggio fondamentale, ed essa resta un argomento invincibile. È un problema aperto, e non un fatto archeologico come spesso vogliono farci credere a teatro. Essa rappresenta da sempre lo scoramento dell'uomo, il suo essere solo su questa terra, il suo confrontarsi con un silenzio immenso che gli sta intorno. Questo è un tema universale, e per chi lavora nel teatro resta una disciplina imprescindibile ${ }^{4}$.

Selon la Socìetas, un théâtre pré-tragique est avant tout infantile. Une théorie de l'expérience du sujet pour la Societas, et en particulier pour Chiara Guidi, reposerait sur une théorie de l'enfance. D'après Annalisa Sacchi (2015), le Teatro infantile de Guidi sera fondamentalement un «théâtre minimal». Non pas comme certaines tendances qui voient dans l'enfance une version réduite

3 «Un langage négatif, le son du langage traduit en haillons et en gravats, qui continue désespérément et urgemment à apporter quelque chose qui doit être communiqué.»

4 «La création de la tragédie est une étape fondamentale pour nous, et elle reste un argument non résolu. C'est un problème ouvert, et non un fait archéologique comme on veut nous le faire croire au théâtre. Elle a toujours représenté le découragement de l'homme, sa solitude sur cette terre, sa confrontation avec l'immense silence qui l'entoure. C'est un thème universel, et pour ceux qui travaillent dans le théâtre, cela reste une discipline essentielle» Fragment du article "Quale teatro per il 2003?», p. 243, Archive de la Societas Raffaello Sanzio (Cesena, Italia). 
de ce qui s'achemine vers l'âge adulte, ni comme une espèce protégée destinée à une minorité, mais bien minimal dans le sens de rendre au théâtre sa forme essentielle.

L'animal, comme l'enfant, se présentent comme des voies d'accès dans cette recherche de l'enfance du théâtre. En effet, tous deux manquent de technique et, par leur présence, nous montrent uniquement qu'ils se trouvent sur scène: il n'y a pas de référent linguistique, car «the animal is pure voice; it has no language: it has a tongue» (Romeo Castellucci en Gabriella Giannachi et Nick Kaye, 2002: 150). La présence de l'enfant et de l'animal sur scène plonge le spectateur dans un temps qui nous est inconnu, voire oublié, qui précède notre entrée dans le langage.

La recherche de ce temps de l'enfance, qui est à son tour le temps du tragique, ne s'oriente pas vers un "retour à» un lieu ou un temps du passé. Ce temps de l'enfance coexiste plutôt avec notre entrée dans le langage, et c'est dans ce temps-là qu'habite l'ineffable, l'incommunicable, ce que nous ne pouvons pas verbaliser, nommer ou conceptualiser. On retrouve ainsi la problématique du sujet face à la prison du langage dans la majeure partie de ces représentations. Celles-ci mettent en exergue cette finitude du mot, soulignant le silence qui l'habite.

Le théâtre de la Raffaello Sanzio entend rompre avec cette vision univoque dans laquelle notre culture occidentale et, plus précisément, l'humanisme européen a placé l'homme et la raison au centre de la pensée hégémonique. Par la présence sur scène d'animaux ainsi que de corps hors norme, ce projet "antihumaniste» vient matérialiser ce même besoin, qu'annonce déjà Giorgio Agamben (2002), en reconnaissant le mystère qui se profile dans notre séparation d'avec l'animal:

Nella nostra cultura, l'uomo è stato sempre pensato come l'articolazione e la congiunzione di un corpo e di un'anima, di un vivente e di un logos, di un elemento naturale (o animale) e di un elemento soprannaturale, sociale o divino. Dobbiamo invece imparare a pensare l'uomo come ciò che risulta dalla sconnessione di questi due elementi e investigare non il mistero metafisico della congiunzione, ma quello pratico e politico della separazione. Che cos'è l'uomo, se esso è sempre il luogo - e, insieme, il risultato - di divisioni e cesure incessanti? Lavorare su queste divisioni, chiedersi in che modo - nell'uomo - l'uomo è stato separato dal nonuomo e l'animale dall'umano, è più urgente che prendere posizione sulle grandi questioni, sui cosiddetti valori e diritti umani. E, forse, anche la sfera più luminosa delle relazioni col divino dipende, in qualche modo, da quella - più oscura - che ci separa dall'animale5. (Agamben, 2002:35-36)

5 «Dans notre culture, l'homme a toujours été pensé comme l'articulation et la conjonction d'un corps et d'une âme, d'un être vivant et d'un logos, d'un élément naturel (ou animal) et d'un élément surnaturel, social ou divin. Il faut plutôt apprendre à penser l'homme comme ce qui résulte de la déconnexion de ces deux éléments et non pas enquêter sur le mystère métaphysique de la conjonction, mais sur le mystère pratique et politique de la séparation. Qu'est-ce que l'homme, s'il est 
Agamben comme la Socìetas aspirent à une réflexion sur ce «mystère pratique» qui s'éloigne de ce «mystère métaphysique » classique de l'union de l'homme avec le divin, du corps avec l'esprit. Son théâtre plonge dans cet "autre» mystère qui nous raconte la séparation, de cette partie sombre de l'être humain, déconnectée de la présence pure. L'apparition des animaux dans les productions théâtrales n'a rien de nouveau, car ils apparaissaient déjà dans les drames liturgiques médiévaux et les productions théâtrales courtoises des $\mathrm{XVI}^{\mathrm{e}}$ et $\mathrm{XVII}^{\mathrm{e}}$ siècles. Cependant, la signification de l'apparition d'animaux dans ces productions est loin d'être celle que le théâtre de la Socìetas veut expérimenter. L'animal

precede l'attore [...]. Il linguaggio è allora connesso al fattore corpo, non lo esprime solamente, ma da esso proviene, non ha più nessuna sintassi, non rimanda a nulla se non alla presenza reale ed immediata di un corpo $^{6}$.» (Ridout, 2002a: 5)

L'animal renvoie à la matérialité pure de l'être et aussi à un noyau pré-tragique et rituel. En ce sens, le corps animal nous rend conscients de notre propre corps animal, de notre présence physique au monde. Bref, l'apparition de l'animal et de l'enfant sur la scène est perçue comme l'irruption du réel dans le fictif, du désinvolte dans l'ordre, de la nature dans la culture.

\section{Le temps du réel}

La réalité, ou plus concrètement la recherche du réel, sera le dernier sommet qui complètera cette forme géométrique. Depuis sa naissance sur la scène iconoclaste dans Santa Sofía, Teatro Khmer (1985), en passant par une réflexion autour du mythe et du tragique dans notre monde contemporain, le souci du réel de la troupe italienne a ouvert un intérêt particulier pour les formes de représentation et leurs limites, et a accordé une attention particulière aux techniques et aux processus, dans la relation de l'art avec son contexte immédiat.

L'évocation du réel à travers une approche illusionniste nous montre que c'est l'artifice même qui dévoile le réel caché dans l'étrange, le mystérique ou le monstrueux. Il révèle un chemin alternatif dans notre vie quotidienne, dans la construction d'un monde parallèle. Cependant, la Socìetas construit ce monde parallèle non seulement à travers l'artifice, mais découvre également dans l'abject l'affirmation du réel en soi. Elle nous présente un corps ouvert, dépourvu

toujours le lieu - et en même temps le résultat - d'incessantes divisions et césures? Travailler sur ces divisions, se demander en quoi - chez l'homme - l'homme a été séparé du non-homme et l'animal de l'humain est plus urgent que de prendre position sur les grandes questions, sur les soi-disant valeurs et droits humains... Et peut-être aussi la sphère la plus lumineuse des relations avec le divin dépend, en quelque sorte, de celle - la plus sombre - qui nous sépare de l'animal.»

6 «L'animal "précède l'acteur [...]. Le langage est alors relié au facteur corps, il ne l'exprime pas seulement, mais il en provient, il n'a déjà plus aucune syntaxe, il ne se réfère à rien sinon à la présence réelle et immédiate d'un corps.» 
d'organes, dans toute sa corporalité. Le corps de l'acteur est présenté comme la limite de la représentation dans une tentative de récupération du réel sur scène.

Un spectacle comme Giulio Cesare (1997) devient un exemple paradigmatique de cette extension vers les limites de la représentation. Giulio Cesare explore les possibilités de la rhétorique comme art de la persuasion, mais à travers la "vérité" des corps. Des corps qui "parlent» d'eux-mêmes, comme lorsqu'apparurent Elena Bagaloni en Brutus ou Cristiana Bertini en Cassius sur scène, deux actrices souffrant d'anorexie en danger réel de mort. En 2014, Romeo Castellucci a fait un remake de Giulio Cesare, mais cette fois seulement à travers des "pezzi staccati», c'est-à-dire deux des monologues centraux de la pièce de 1997: le discours du personnage ...vskij - écho du père fondateur du théâtre du $\mathrm{xx}^{\mathrm{e}}$ siècle, Stanislavskij - et l'oraison funèbre de Marc Antoine en l'honneur de César. Le premier est récité par un interprète qui porte une caméra endoscopique dans sa gorge, nous montrant presque ce corps sans organes; le second est joué par un acteur laryngectomisé. La caméra endoscopique renvoie sur un écran circulaire l'image de l'appareil vocal de ...vskij, c'est-à-dire les mouvements de la glotte et des cordes vocales. L'instrument vocal d'Antonio est mutilé et sa voix atteint le spectateur amplifiée et altérée par un appareil. Ce qui nous est montré sur scène, c'est la présence du corps déformé, souffrant ou atypique; un corps qui vient d'être traité comme un corps-sculpture (Lehmann, 2013).

Le corps est déformé ou plus précisément est montré dans sa déformation. Le corps lui-même est un objet, un objet transitionnel (Winnicott, 1993), qui nous fascine et qui devient à son tour un sujet. C'est ce sujet qui stimule le sentiment que nous-mêmes ne sommes pas seulement des sujets vivants, mais aussi en partie des objets. La frontière s'estompe à nouveau et nous restons plongés dans le liminal quand «le sujet tend à être une chose et la chose un être vivant, quand se perd la sécurité de pouvoir séparer avec certitude la vie et la mort, le sujet et l'objet» (Lehmann, 2013: 367). Les scènes de Romeo Castellucci sont aux limites du «normal» - du social, selon Turner (1969) -, elles sont dans ces états de liminalité où le corps, en suivant Lehmann (2013), devient «inhumain ». Mais elles vont plus loin, puisque ces corps évitent toute catégorisation et, paradoxalement, permettent à la beauté du corps dans sa déformation de devenir visible et, en même temps, font de l'organique une expérience d'une beauté fascinante.

La seconde forme de représentation du réel que la troupe a abordée durant toutes ces années fait du relationnel un véhicule d'expression artistique. Son esthétique ne pouvait se limiter à un travail sur la scène théâtrale. La troupe devait donc étendre son champ de vision à la réalité ambiante et mener ainsi ses recherches dans un contexte plus immédiat. Le relationnel devient donc un travail sur le réel.

Depuis les années 90, les propositions artistiques montrent un nouveau tournant vers le social qui approfondit encore plus le caractère performatif des pièces et promeuvent le nouveau paradigme du spectateur. Ce nouveau tournant 
travaille sur l'aspect social des pièces de théâtre, développant différentes formes artistiques (Nicolas Bourriaud, 2001 et Claire Bishop, 2012). L'essence de cette pratique artistique réside dans l'invention de relations entre les sujets, où chaque œuvre d'art nous permet de créer un faisceau de relations avec le monde, qui à leur tour créent d'autres relations, et ainsi de suite. À l'instar de la scène théâtrale, les Scuola de Claudia Castellucci se transforment aussi en un modèle qui s'éloigne de la réalité "quotidienne», et nous invite à créer un autre instant, à habiter un autre espace-temps, parallèle à cette réalité que nous connaissons déjà.

Depuis les années 90, Claudia Castellucci a créé différentes écoles, toutes consacrées à l'étude du rythme (Stoa, Calla, Mora, Ritmo drammatico) et de la représentation (Scuola theatre della discesa, Scuola Cònia, Setta. Scuola di tecnica drammatica). Pour Castellucci, le concept de Scuola est étroitement lié à la perception du temps et, plus précisément, de la durée. La Scuola devient. Il y a une idée très claire au début, c'est ce que Castellucci matérialise comme une structure - ou, pour reprendre les mots de l'italienne, un schéma - et en même temps cette idée est susceptible de métamorphose, un changement dérivé de la rencontre entre les personnes qui en font partie. La Scuola serait donc un être ensemble d'une manière déterminée et, en conséquence, acquérant une position dans le monde. Pour Jacques Rancière, ce positionnement, cette cohabitation concrète, se constitue comme l'axiome d'un art politique:

Si l'art est politique, ce n'est ni par les messages qu'il transmet sur l'ordre du monde, ni par la manière dont il représente les structures et les conflits sociaux, c'est par la manière dont il découpe le temps et peuple l'espace. (Rancière, 2004:37)

L'origine étymologique du mot «Scuola» vient du terme latin scola (ou schola), lui-même dérivé du grec $\sigma \chi 0 \lambda \eta \dot{~(s c h o l e ̀) ~ e t ~ q u i, ~ c o n t r a i r e m e n t ~ a ̀ ~ c e ~ q u e ~ n o u s ~}$ pourrions penser aujourd'hui, signifiait loisirs et repos. Le «scholè» était exactement le moment où l'on se reposait des efforts de la vie quotidienne pour se consacrer à l'étude, à la raison. Ce n'est que plus tard, avec la naissance de la première école publique sous l'empire de Carlo Magno, que le terme sera prolongé pour indiquer le lieu de rencontre des enseignants et des élèves. Claudia Castellucci est consciente de la charge étymologique que porte le mot "scuola». Pour cette raison, elle l'applique à sa création artistique comme modèle de distance avec la réalité quotidienne, dans le but de générer un autre temps et d'habiter un autre espace, parallèle à cette réalité que nous connaissons déjà.

\section{Considération finale: Le temps du spectateur}

Quel temps habite le spectateur de la Societas Raffaello Sanzio? Le travail de la Societas remet en cause la forme même de la représentation théâtrale, et par extension, du monde. Il nous interroge sur le regard que porte le spectateur, et la possibilité de créer une mythologie contemporaine qui questionne les 
problématiques actuelles de l'être (ou du devenir) spectateur. Romeo Castelluci expliquait:

Penso che un'esperienza teatrale debba essere fondata su un certo tipo di fatica, di "stress" - che è la somma del piacere -. Lo spettatore, cioè, dovrebbe essere abbandonato davanti ad alcuni problemi gettati davanti al suo volto. Lo spettatore dovrebbe essere lasciato da solo a confrontarsi con nuovi conflitti, testimone oculare del suo stesso essere spettatore, perché guardare qui significa essere visti dall'immagine ed essere messi a nudo da essa. Si tratta cioè di guardarsi guardare, di vedere il proprio sguardo. L'immagine a teatro specchia la maschera del tuo volto, la tua solitudine, che qui è trattata, trasfigurata, riconciliata ${ }^{7}$. (Romeo Castellucci, 2016: 315)

La disparition de l'artiste créateur que l'on peut qualifier d'abandon au spectateur, implique une cession de l'espace, la possibilité de «se regarder regarder», d'être touché. Autrement dit de nous arrêter dans cet espace qui offre un moment fondamental d'immersion réflexive, une interruption temporaire de notre perception habituelle du monde et des logiques qui le contiennent. Elle place le spectateur dans un lieu à risque qui le met complètement à nu, et le précipite dans un espace de désorientation et d'imprévu. Cette nouvelle perspective est intérieure, invisible, traverse avec fulgurance ces trois sommets du triangle et crée un nouvel espace/temps commun et profondément politique. Un espace/ temps dans lequel les notions d'émancipation et de transformation de la réalité acquièrent un poids fondamental. Toutes deux nous permettent de déplacer nos attentes vers un lieu distinct, et peut-être de sortir en tant qu' "autres" par la même porte par laquelle nous sommes entrés. Le travail de la Societas constitue donc un projet à la fois esthétique et éthique, dans la mesure où il cherche à ouvrir des brèches dans la notion classique de représentation, tout en nous offrant d'autres formes de représentation de notre monde et de nous-mêmes.

\section{Bibliographie}

Agamben, G. (2002), L'aperto. L'uomo e l'animale, Milano: Bollati Boringhieri Bishop, C. (2012), Artificial Hells. Participatory Arts and the Politics of Spectatorship, New York, Verso Books

Bourriaud, N. (2001), Esthétique relationnelle, Dijon: Les Presses du réel.

7 «Je crois qu'une expérience théâtrale doit reposer sur un certain type de fatigue, de "stress": l'addition du plaisir. C'est-à-dire que l'on devrait laisser le spectateur livré à lui-même face aux difficultés qu'il rencontre. Il reviendrait à ce dernier d'affronter seul de nouveaux conflits: être le témoin oculaire de son propre être spectateur. Regarder ici signifie être vu à travers l'image et y rester exposé. Il s'agit, en d'autres termes, de se regarder regarder, observer son propre regard. L'image théâtrale reflète le masque de notre visage et de notre solitude, afin d'être examinée, transfigurée, réconciliée.» 
Calchi, G. (2009), "Language Under Attack: The Iconoclastic Theatre of Societas Raffaello Sanzio", Theatre research international, vol. 1, n. 34, p. 50-65.

Campbell, Joseph (2015 [1988]). El poder del mito, traducción César Aira, Madrid: Capitán Swing.

Castellucci, C., Castellucci, R., Guidi, C., Kelleher, J., Ridout, N. (2007), The Theatre of Societas Raffaello Sanzio, New York, Roudlegde.

Castellucci, Claudia (2015). Setta. Scuola di tecnica drammatica, Macerata, Quodlibet.

Castellucci, C., Castellucci, R. (1992), II teatro della Societas Raffaello Sanzio. Dal teatro iconoclasta alla super-icona, Milano, Ubulibri.

Castellucci, C. (2001), Les pèlerins de la matière. Théorie et praxis du théâtre. Écrits de la Societas Raffaello Sanzio, texte italien (Italie) traduit par Karin Espinosa, Besançon, Les Solitaires Intempestifs

Castellucci, R. (2007), Tragedia endogonidia, de Romeo Castellucci y la Socìetas Raffaello Sanzio, dirigido por Romeo Castellucci. [Once episodios. Cesena, Avignon, Berlin, Bruxelles, Bergen, Paris, Roma, Strasbourg, London, Marseille, Cesena] Video Cristiano Carloni y Stefano Franceschetti, música Scott Gibbons, 3 DVD, 1 CD, 86-page booklet.

Castellucci, Romeo (2008), Itinera. Trajectoires de la forme. Tragedia Endogonidia, traducción de J.-L. Provoyeur, Arles, Actes sud.

Eiermann, André (2012), “Teatro postespectacular. La alteridad de la representación y la disolución de las fronteras entre las artes”, traducción Micaela van Muylem, Telón de Fondo. Revista de teoría y crítica teatral, 16 (diciembre 2012), disponible en www.telondefondo.org (consultado el 15 de septiembre de 2018), capítulo inicial del libro Postspektakuläres Theater. Die Alterität der Aufführung und die Entgrenzung der Künste (Bielefeld, transcript Verlag, 2008).

Eliade, Mircea (1998 [1957]), Lo sagrado y lo profano, traducción de L. Gil Fernandez y R. A. Díez Aragón, Barcelona, Paidós.

Fischer-Lichte, Erika (2011 [2004]), Estética de lo performativo, traduction Diana González Martín et David Martínez Perucha, prologue Óscar Cornago, Madrid, Abada Editores.

Grotowski, Jerzey (2002), Towards a Poor Theatre, Ed. Eugenio Barba et prologue Peter Brook, New York, Routledge.

Lehmann, Hans-Thies (2013 [1999]), Teatro posdramático, traduction Diana González Martín, Murcia, Cendeac.

Lehmann, Hans-Thies (2017 [2014]), Tragedia y teatro dramático, traduction Claudia Cabrera, Méxido D. F, Paso de Gato.

Rancière, Jacques (2004). Malaise dans l'esthétique, Paris, Galilée.

Ridout, N. (2002), “B.\#03, Perché, dunque, lei vive”, Idioma, Clima, Crono. Quaderni dell'Itinerario. Tragedia Endogonidia, C. Astrié, J. Kelleher et N. Ridout (eds.), 6 volumi, Cesena. 
Ridout, Nicholas (2002a). "Fuori nell'Aperto". Idioma, Clima, Crono IV, 6 volumi, Cesena.

Schechner, Richard (2002). Performance studies. An introduction, New York, Routledge. 